\title{
Psychiatric morbidity and suicidal behaviour in psoriasis
}

DOI:

10.1111/bjd.17004

\section{Document Version}

Accepted author manuscript

Link to publication record in Manchester Research Explorer

\section{Citation for published version (APA):}

Parisi, R., Webb, R. T., Kleyn, C. E., Carr, M. J., Kapur, N., Griffiths, C. E. M., \& Ashcroft, D. M. (2019). Psychiatric morbidity and suicidal behaviour in psoriasis: a primary care cohort study. The British journal of dermatology, 180(1), 108-115. https://doi.org/10.1111/bjd.17004

\section{Published in:}

The British journal of dermatology

\section{Citing this paper}

Please note that where the full-text provided on Manchester Research Explorer is the Author Accepted Manuscript or Proof version this may differ from the final Published version. If citing, it is advised that you check and use the publisher's definitive version.

\section{General rights}

Copyright and moral rights for the publications made accessible in the Research Explorer are retained by the authors and/or other copyright owners and it is a condition of accessing publications that users recognise and abide by the legal requirements associated with these rights.

\section{Takedown policy}

If you believe that this document breaches copyright please refer to the University of Manchester's Takedown Procedures [http://man.ac.uk/04Y6Bo] or contact uml.scholarlycommunications@manchester.ac.uk providing relevant details, so we can investigate your claim.

\section{OPEN ACCESS}


DR ROSA PARISI (Orcid ID : 0000-0002-0968-9153)

Article type : Original Article

\section{Psychiatric morbidity and suicidal behaviour in psoriasis:}

\section{a primary care cohort study}

Running Head: Psychiatric morbidity and suicidality behaviour in psoriasis

R. Parisi ${ }^{1}$, R.T. Webb ${ }^{2,5}$, C.E. Kleyn ${ }^{3,4}$, M.J. Carr ${ }^{2}$, N. Kapur ${ }^{2,5}$, C.E.M. Griffiths ${ }^{3,4}$, D.M. Ashcroft ${ }^{1,4,5}$

${ }^{1}$ Centre for Pharmacoepidemiology and Drug Safety, School of Health Sciences, University of Manchester, Manchester Academic Health Science Centre (MAHSC), Manchester, UK; ${ }^{2}$ Centre for Mental Health and Safety, School of Health Sciences, University of Manchester, Manchester Academic Health Science Centre (MAHSC), Manchester, UK; ${ }^{3}$ Dermatology Centre, Salford Royal NHS Foundation Trust, University of Manchester, Manchester Academic Health Science Centre (MAHSC), Manchester, UK; ${ }^{4}$ NIHR Manchester Biomedical Research Centre, Faculty of Biology, Medicine and Health, The University of Manchester, Manchester, UK; ${ }^{5}$ NIHR Greater Manchester Patient Safety Translational Research Centre, University of Manchester, Manchester Academic Health Science Centre (MAHSC), Manchester, UK

This article has been accepted for publication and undergone full peer review but has not been through the copyediting, typesetting, pagination and proofreading process, which may lead to differences between this version and the Version of Record. Please cite this article as doi: 10.1111/bjd.17004

This article is protected by copyright. All rights reserved. 


\section{Corresponding author: Dr Rosa Parisi}

E-mail: rosa.parisi@manchester.ac.uk

Funding source: The research was funded by Salford Royal NHS Foundation Trust in conjunction with the National Institute for Health Research (NIHR) Programme Grant: Identification and Management of Psoriasis Associated ComorbidiTy (IMPACT) (RP-PG-0608-10163). The views expressed are those of the authors and not necessarily those of the NHS, the NIHR or the Department of Health. The funders had no role in study design, data collection and analysis, decision to publish, or preparation of the manuscript. CEMG and DMA are funded in part by the Medical Research Council (MR/L011808/1). CEMG is an NIHR Senior Investigator.

This study is based in part on data from the Clinical Practice Research Datalink (CPRD) obtained under licence from the UK Medicines and Healthcare products Regulatory Agency. The data is provided by patients and collected by the NHS as part of their care and support. The Office for National Statistics (ONS) is the provider of the ONS Data contained within the CPRD. Hospital Episode Data and the ONS Data Copyright (C (2014), are re-used with the permission of The Health \& Social Care Information Centre. All rights reserved. The study was approved by the independent scientific advisory committee (ISAC) for Clinical Practice Research Datalink research (reference number: 15_033R). The interpretation and conclusions contained in this study are those of the authors alone.

Clinical Practice Research Datalink data cannot be shared because of licensing restrictions (https://www.cprd.com/ISAC/).

Conflict of interest: The authors state no specific conflicts of interest relevant to this study.

This article is protected by copyright. All rights reserved. 
CEK has received honoraria and/or research funding from Celgene, Pfizer, Janssen, Johnson \& Johnson and honoraria from Abbvie, Amgen, Galderma, Leo Pharma and GSK-Stiefel, Eli Lilly. DMA has received research grants from Abbvie and the Leo Foundation and has had personal fees from Pfizer and GSK. CEMG has received honoraria and/or research grants from Abbvie, Actelion, Almirall, Amgen, Celgene, Galderma, LEO Pharma, Eli Lilly, GSK-Stiefel, Janssen, MSD, Novartis, Pfizer, Sandoz, Sun Pharmaceuticals and USB Pharma.

\section{What's already known on this topic?}

- Psychosocial comorbidity is common in patients with psoriasis. Earlier studies have tried to examine risk of suicide but limited by power to examine risk of this rare outcome

\section{What does this study add?}

- The overall risk of suicide in people with psoriasis was $40 \%$ lower compared to peers without the condition. The risk of suicide varied according to age: it was $60 \%$ lower among individuals with psoriasis diagnosed at $\geq 40$ years compared to people without the disease of the same age group, whereas if the diagnosis occurred prior to age 40 years there was no difference in risk between the two groups.

- Conversely, there was $15 \%$ increase in self-harm risk in the psoriasis group versus the general population

This article is protected by copyright. All rights reserved. 


\section{ABSTRACT}

Background: Psychological distress among people with psoriasis may lead to elevated risks of suicide and non-fatal self-harm.

Objectives: To investigate psychiatric comorbidity, psychotropic medication prescribing and risk of suicidality in people with psoriasis.

Methods: A cohort of patients with psoriasis (1998-2014) was delineated using the Clinical Practice Research Datalink, with linkage to Hospital Episode Statistics and Office for National Statistics mortality records. Each patient with psoriasis was matched with up to 20 patients without psoriasis on age, gender, and general practice. A stratified Coxregression model was used to estimate the hazard ratios (HRs) for suicide or non-fatal self-harm risks adjusted for socioeconomic status.

Results: At baseline, among 56,961 and 876,919 patients with and without psoriasis, higher prevalence for histories of alcohol misuse, bipolar disorder, depression, anxiety disorders, self-harm, and psychotropic drug prescription were observed. The deprivation-adjusted hazard ratio indicated lower suicide risk inpeople with psoriasis $(0.59,0.41-0.85)$.The risk of suicide varied according to age: it was lower in people with psoriasis diagnosed at $\geq 40$ years ( $H R 0.38,0.21-0.66$ ), whereas there was no difference in risk of suicide in people with psoriasis diagnosed before 40 years (HR 0.92, 0.581.46).Conversely, there was a small increased risk for self-harm (HR 1.15, 1.04-1.27) associated with psoriasis.

Conclusions: The prevalence of mental illness was raised in people withpsoriasis and this may lead to a greater risk of self-harm. Nevertheless, having psoriasis does not appear to be associated with an increased risk of suicide. Healthcare professionals caring for patients with psoriasis should continue to monitor and tackle effectively the psychological needs of these individuals.

This article is protected by copyright. All rights reserved. 


\section{INTRODUCTION}

Psoriasis is a currently incurable, chronic skin disorder. It is among the most common immunemediated diseases ${ }^{1}$ affecting almost $3 \%$ of people in the UK ${ }^{2}$. The condition is commonly associated with psychiatric comorbidities ${ }^{3-7}$. Suggested explanations for associated mental illness include the considerable impact that psoriasis has on people's quality of life ${ }^{8,9}$, mainly related to the appearance of the skin and consequent psychological challenges ${ }^{10}$. It is thought that the psychological burden in people with psoriasis may lead to elevated risk of self-harm and suicide.

Several earlier studies have examined possible associations between psoriasis and suicidal ideation $^{3,5,11}$, but few population-based studies have been published ${ }^{4,6,12-14}$. Previous studies have investigated composite outcomes (such as suicidal ideation, suicide attempt or suicide combined) (4,6 $^{4}$ or have identified people with psoriasis from hospital records ${ }^{12}$, insurance and administrative databases $s^{6,14}$, or have used a hospital register linked to dispensing data ${ }^{13}$. Two recent systematic reviews and meta-analyses examined suicidality risk in people with psoriasis ${ }^{15,16}$ and reached different conclusions. Singh et al. ${ }^{15}$ identified 18 studies (including cross-sectional, case-control and cohort studies) that reported on suicidal ideation, attempted suicide or death by suicide. They concluded that people with psoriasis have a raised prevalence of suicidal ideation and elevated risks of both fatal and non-fatal suicidal acts. Conversely, Chi et al. ${ }^{16}$ investigated the same outcomes but included only four population-based cohort studies, given these studies are more appropriate to assess the causal relationship between psoriasis and the outcome ${ }^{16}$. They concluded that there was insufficient evidence of an association between psoriasis and suicidality risk, and they also highlighted the need for further research on this important topic ${ }^{16}$.

This article is protected by copyright. All rights reserved. 
Given the lack of clarity regarding this clinically important issue, we conducted a large populationbased cohort study utilising linked primary care, hospitalisation and national mortality records to investigate the prevalence of psychiatric comorbidities, psychotropic medication prescribing and the associations between psoriasis and suicide and self-harm risks.

\section{MATERIALS AND METHODS}

\section{Data Source and Study Design}

A population-based cohort study was carried out using data from the Clinical Practice Research Datalink (CPRD) linked to the Hospital Episode Statistics (HES) and Office for National Statistics (ONS) mortality records. The study is reported in line with the recommendations of the RECORD statement ${ }^{17}$.

Virtually the entire population of the UK is registered with a general practice, which plays a key role in the National Health Servive (NHS). The CPRD, one of the world's largest longitudinal primary care databases, captures the medical histories (diagnoses, test results, prescriptions and referrals to hospital) of patients registered with a general practice. Published studies have shown that the CPRD yields high-quality data for conducting observational research studies ${ }^{18,19}$. The current study included data from 398 general practices in England that had consented to linkage to national HES and ONS mortality records. Approximately $60 \%$ of all practices based in England are included in the CPRD's routine linkage scheme, with linkage undertaken using patients' unique NHS identification numbers, and available information on gender, date of birth and residential postcode.

The study cohort consisted of patients with a first diagnosis of psoriasis recorded using Read codes in their primary care records between $1^{\text {st }}$ January 1998 and $31^{\text {st }}$ March 2014 who were 16 years or older at index date, who had been registered with the practice for at least one year prior to index date, and who were eligible for linkage to HES and to ONS mortality records. A comparison cohort of

This article is protected by copyright. All rights reserved. 
patients without psoriasis was identified by matching each patient with psoriasis with up to 20 patients without the disease on age, gender and general practice on index date ${ }^{20}$. To avoid potential selection, information and detection biases, patients in the comparison cohort were selected from the same population at risk and were identified from the same general practice and during the same time window of the psoriasis patients they were matched to. This delineated the initial cohort used to investigate the association between psoriasis and suicide risk (Supplementary Fig. 1).

Subsequently from this initial cohort, people with a history of self-harm (at index date) in either CPRD or HES were excluded from the analysis. This nested cohort was used to investigate the association between psoriasis and the risk of self-harm. In the analysis of both outcomes, all patients included in the study were followed-up from index date until the end of the study ( $31^{\text {st }}$ March 2014) or the last date of data collection or the patient was transferred out of the practice, died or experienced one of the outcomes of interest (first non-fatal self-harm event or died by suicide), whichever came first.

\section{Area-level Socioeconomic Status}

Information on small area-level socioeconomic status was available through linkage of patients' residential postcodes to the Index of Multiple Deprivation (IMD) $2010^{21}$. The IMD is an ecological measure that is derived via a weighted combination of the following seven domains of deprivation: income, employment, health and disability, education skills and training, barriers to housing and services, living environment, and crime. The IMD scores were aggregated into quintiles, where 1 corresponded to the least deprived areas and 5 to the most deprived localities.

This article is protected by copyright. All rights reserved. 


\section{Classification of Suicide and Non-fatal Self-harm}

People who died by suicide were ascertained using the underlying cause of death as recorded on the death certificate. These were extracted from the linked ONS mortality records using the following International Classification of Diseases codes, Ninth Revision (ICD-9) (E950-E959; E980-E989 excluding E988.8) and International Statistical Classification of Diseases and Related Health Problems, Tenth Revision (ICD-10) codes (X60-X84, Y10-Y34 - excluding Y33.9, Y87.0, Y87.2). The definition of suicide applied was in line with the guidance issued by the ONS, which combines cases where the underlying cause of death was either suicide or an unnatural death of undetermined intent $^{22,23}$. Unnatural causes of death are returned in England \& Wales by a coroner; psychiatrists may provide evidence and expert professional judgement at coronial hearings, but the cause of death is ultimately determined by the coroner. First-recorded non-fatal self-harm episodes were identified according to first entry in either the primary care or hospital records. Read codes were used to identify events from the primary care records ${ }^{24}$, and ICD-9 (E950-E959) and ICD-10 (X60-X84) codes, to identify events from HES. All of the code lists used in the study are available from www.clinicalcodes.org ${ }^{25}$.

\section{Statistical Methods}

Prevalence ratios (PRs) for psychiatric comorbidities and psychotropic drug prescription at baseline were calculated using a fixed-effect Poisson regression model. All-persons and gender-specific suicide and self-harm incidence rates per 10,000 person-years, with their $95 \%$ confidence intervals (Cls), were calculated for patients with and without psoriasis. Cox proportional hazard models stratified according to matched set were fitted to estimate hazard ratios (HRs) (95\% Cls) for suicide and self-harm in people with psoriasis versus the comparison cohort. The models were adjusted for area-level socioeconomic status measured by the IMD score. Additional analyses examining the risk of self-harm by psoriasis severity were performed. Here, the severity of psoriasis was modelled as a

This article is protected by copyright. All rights reserved. 
time-varying covariate based on systemic treatment exposure (acitretin, etretinate, ciclosporin, hydroxycarbamide, methotrexate, fumaric acid, phototherapy or a biologic therapy). For both outcomes, interactions between psoriasis and age, gender and IMD quintile were tested separately.

To assess the robustness of the results, several sensitivity analyses were performed for both outcomes, as follows: i) to explore the possibility of detection bias, the analysis was restricted to patients who consulted at least once a year during follow-up; ii) the statistical model was rerun with the psoriasis cohort restricted to individuals without psoriatic arthritis; iii) the suicide definition was altered by excluding unnatural deaths of undetermined event. All statistical analyses were performed using Stata v14.

\section{RESULTS}

\section{Demographics}

The cohort for examining suicide included 56,961 patients with incident psoriasis diagnoses and 876,919 patients in the comparison group. Overall, there were more women (55.3\%) than men; median (interquartile range, IQR) age at index date was 46 (28) years old (Table 1). Nested within this cohort, a slightly smaller study cohort for examining non-fatal self-harm as the outcome was also delineated, restricted to people without a history of self-harm prior to index date, including 54,709 patients with psoriasis and 813,699 comparison patients. At baseline, $688(1.3 \%)$ patients with psoriasis had received phototherapy, systemic or biologic therapies which increased to 2,491 (4.6\%) by the end of follow-up (Supplementary Table 1).

This article is protected by copyright. All rights reserved. 


\section{Mental Illness Diagnoses and Psychotropic Medication Prescribing}

As shown in Table 2, compared to the psoriasis-free cohort at baseline, patients with psoriasis had higher prevalence ratios (PRs) for histories of alcohol misuse (PR 1.30, 95\% Cl 1.23-1.37), bipolar disorder (PR 1.23, 1.10-1.37), depression (PR 1.17, 1.15-1.20), anxiety disorders (PR 1.15, 1.13-1.18), self-harm (PR 1.10, 1.06-1.15), and psychotropic medication prescription (PR 1.13, 1.12-1.14). The prevalence ratios for histories of alcohol misuse, mental illnesses and psychotropic medication prescription were lower but had a similar distribution when removing people with a history of selfharm.

\section{Suicide and non-fatal self-harm risk}

During a median (IQR) of 4.4 (6.1) years of follow-up, 35 and 679 suicide deaths occurred in the psoriasis and comparison cohort, respectively. Having excluded patients with a history of self-harm, 470 self-harm events occurred in the psoriasis cohort and 5,281 of these events occurred in the comparison group, respectively. Suicide rates were lower in the psoriasis cohort $(1.1 / 10,000$ personyears) than the comparison cohort (1.5/10,000 person-years), whereas, self-harm rates were higher in the psoriasis $(15.0 / 10,000$ person-years) than the comparison cohort $(12.4 / 10,000$ person-years), Table 3. Results from the Cox proportional hazards analysis showed that suicide risk in patients with psoriasis was significantly lower than the comparison cohort having accounted for socioeconomic status (adjusted HR 0.59, 0.41-0.85). After excluding patients with a history of self-harm, there was a small and significant elevation in risk of self-harm among patients with psoriasis (HR 1.15, 1.04-1.27) (Table 4). Severe psoriasis was associated with a non-significantly elevated risk of self-harm HR 1.11, 0.63-1.97 (Supplementary Table 3). An interaction test revealed a variation in suicide risk by age $(p=0.017)$. The hazard ratio for suicide was $0.92(0.58-1.46)$ for people with psoriasis diagnosed when they were younger than 40 years, whereas the HR was $0.38(0.21-0.66)$ for people with psoriasis diagnosed at 40 years and above, Figure 1 . There was no significant evidence of effect

This article is protected by copyright. All rights reserved. 
modification by gender $(p=0.539)$ or by socioeconomic status $(p=0.827)$. Similarly, no significant interactions were found in the cohort for examining self-harm between psoriasis and age $(p=0.736)$, gender $(p=0.918)$ or socioeconomic status $(p=0.255)$.

\section{Sensitivity analyses}

For the suicide analysis, similar results were obtained when the analysis was restricted to patients who visited their GP at least once a year or when we excluded unnatural deaths of 'undetermined intent' from the suicide definition. The hazard ratio for suicide slightly increased when patients with psoriatic arthritis were removed from the cohort, although it remained below unity and significantly so (Table 4). Conversely, when the same set of sensitivity analyses was conducted for the examination of self-harm as the outcome the results of the primary analysis were confirmed when people with psoriatic arthritis were excluded. However, when the cohort was restricted to patients who visited the practice at least once a year, the risk of self-harm remained slightly increased but no longer significantly so (HR 1.10, 0.98-1.22).

\section{DISCUSSION}

\section{Main Findings}

Our large population-based cohort study has found lower suicide risk among people diagnosed with psoriasis versus individuals of the same age, gender and general practice without the condition.

There was evidence of effect modification with age; specifically, the risk of suicide was lower among individuals diagnosed with psoriasis when they were 40 years or older compared to people without the disease of the same age group, whereas if the diagnosis occurred prior to age 40 years there was no difference in risk versus the matched comparison group.

This article is protected by copyright. All rights reserved. 
At the time of diagnosis, people with psoriasis had a higher prevalence for histories of alcohol misuse, bipolar disorder, depression, anxiety disorders, self-harm, and were more likely to have been prescribed with psychotropic medication. Following diagnosis, we found a small elevation in self-harm risk in the psoriasis group versus the general population, and the risk of self-harm associated with severe psoriasis was also elevated but not significantly so. The robustness of our findings were assessed in a series of sensitivity analyses. When the analysis was restricted to people without psoriatic arthritis, the hazard ratio for suicide remained below unity. For the self-harm analysis, the results remained largely unchanged except for when the cohort was restricted to people visiting the GP at least once a year, where the risk of self-harm associated with psoriasis was slightly elevated but no longer significantly so.

\section{Strengths and Limitations}

The study had several key strengths. First, this was a very large population-based cohort study of more than 50,000 patients diagnosed with psoriasis that was well-powered to investigate suicide as a rare cause-specific mortality outcome. The large cohort sizealso enabled detailed exploration of effect modification by age, gender and area-level socioeconomic status. Importantly, the many number of comparison patients matched to each individual with psoriasis (1:20), generated precise estimates with narrow confidence intervals ${ }^{20}$. Second, risks for fatal and non-fatal self-harm were analysed as two discrete outcomes in the same cohort at risk, which is advantageous in understanding the two phenomena separately ${ }^{26}$. Third, linking primary care data with hospital records and mortality records enabled ascertainment of additional self-harm and suicide events than using the CPRD primary care records alone ${ }^{27}$, an approach that would have underestimated self-harm incidence by over $50 \%{ }^{27}$. Fourth, the psoriasis cohort examined in this study was 
identified from a routinely collected primary care database. Therefore our findings are likely to be more representative of all patients diagnosed with psoriasis in the population compared to studies where the psoriasis cohort was identified solely from hospital episodes. Some limitations of the study should also be considered in interpreting our findings. First, given that GPs do not routinely record standardised information on disease severity, such as the PASI score, the classification of severity of psoriasis according to prescribed medication presented challenges given that these treatments may have other indications ${ }^{28}$. Second, although we examined risk of self-harm according to psoriasis severity, it was not possible to investigate the risk of suicide by severity of psoriasis due to the fact that severe psoriasis has a much lower prevalence and suicide is a very rare outcome. Third, although we exploited linkage between primary and secondary care data to enhance detection of self-harm events, it is likely that some events may not have been ascertained as some patients do not present to general practice or hospitals following selfharm $^{27}$. Finally, despite the steps taken to minimise surveillance bias, the raised prevalence of psychiatric comorbidities and psychotropic medication prescribing that we observed versus persons without psoriasis might be due to affected individuals seeking care more often.

\section{Comparison with existing evidence}

Comparison with other studies is challenging due to differing outcome definitions applied, such as whether or not self-harm and suicide were combined or analysed as discrete endpoints. Wu et al. ${ }^{6}$ utilised an administrative database from the US and reported a raised prevalence of depression (IRR 1.14, 1.11-1.17) in people with psoriasis. Using hospital records from England, Singhal et al. ${ }^{12}$ investigated self-harm and suicide in patients with comorbid mental and physical illnesses. They found only a borderline significant association between psoriasis and suicide risk (rate ratio, RR 1.3,

This article is protected by copyright. All rights reserved. 
1.0-1.8) and evidence of greater self-harm risk associated with psoriasis compared to the general population (RR 1.6, 1.5-1.7). More recently, a study from Denmark examined both non-fatal selfharm and suicide in patients with psoriasis from a large population-based study of more than 400,000 individuals $^{13}$. The study showed no increased suicide risk in mild (IRR 1.05, 0.84-1.32) or severe psoriasis (IRR $0.78,0.45-1.36$ ) whereas they found a significant increased risk of self-harm associated with severe psoriasis IRR 1.69 (1.00-2.84). Our findings are not directly comparable to those of Wu et al. ${ }^{6}$ and Kurd et al. ${ }^{4}$ who, using a primary care database from the UK, reported an increased risk of suicidality, given that both studies investigate the risk of suicidality in people with psoriasis as a combined outcome (fatal and non-fatal suicide attempt). The main methodological differences between our cohort study and previously published investigations are as follows: 1) Varying outcome definitions (e.g. suicide and non-fatal self-harm analysed separately vs. combined as a single outcome variable ${ }^{4,6} ; 2$ ) Use of linked primary care, secondary care and mortality records, rather than primary care ${ }^{4}$ or secondary care ${ }^{12}$ alone, to enhance detection of outcomes of interest; 3) Identification of the psoriasis population from primary care rather than secondary care ${ }^{12}$ as being hospitalised for a major physical illness per se is linked with increased risk of suicide ${ }^{29}$. It is also noteworthy that the ratio of non-fatal self-harm to suicide events found in our study is in line with that reported Singhal et al. ${ }^{12}$, but does not concur with the Danish study which indicates a higher number of suicides events compared to self-harm events ${ }^{13}$. Due to the classification system used, that study is likely to have captured only around a tenth of all the self-harm events that occurred in Denmark $^{26,30}$.

\section{Interpretation}

Suicide and self-harm risks are complex behaviors influenced by many social, psychological and environmental factors. The findings indicate that healthcare professionals should be aware of the psychological burden people with psoriasis carry by monitoring psychiatric comorbidities. This higher prevalence of psychiatric comorbidities, may lead to a small elevation in self-harm risk associated

This article is protected by copyright. All rights reserved. 
with people affected by psoriasis. Interestingly, our findings showed a significantly reduced risk of suicide among people with psoriasis who were diagnosed at 40 years or older. Psoriasis has been classified as type I and II psoriasis according to whether the diagnosis occurs before (type I) or after 40 years old (type II) ${ }^{31}$ and these are often considered as two distinct disorders with different clinical and genetic characteristics ${ }^{32}$. One possible explanation for the inverse relationship that we observed between type II psoriasis and suicide risk may be explained by competing risks for other causes of death. For instance, we have recently reported on an elevated risk of alcohol-related mortality ${ }^{33}$. Furthermore, our findings show that patients with psoriasis are treated more often for psychiatric comorbidities, and so their mood-related disorders, and consequently their suicide risk, may also be better monitored, treated and controlled than in persons without psoriasis.

\section{Author Contribution}

Conceived and designed the study: RP, RTW, DMA. Statistical analysis: RP, MJC. Wrote the first draft of the manuscript: RP. Edited the manuscript: all authors. Interpretation of findings: all authors. Agree with the manuscript's results and conclusions: all authors. RP had full access to all the data in the study and takes responsibility for the integrity of the data and the accuracy of the data analysis.

This article is protected by copyright. All rights reserved. 


\section{REFERENCES}

1 Griffiths CEM, Barker JNWN. Pathogenesis and clinical features of psoriasis. Lancet 2007; 370: 263-71.

2 Springate DA, Parisi R, Kontopantelis E et al. Incidence, prevalence and mortality of patients with psoriasis: a U.K. population-based cohort study. Br J Dermatol 2017; 176: 650-8.

3 Gupta M, Gupta A. Depression and suicidal ideation in dermatology patients with acne, alopecia areata, atopic dermatitis and psoriasis. Br J Dermatol 1998; 139: 846-50.

$4 \quad$ Kurd SK, Troxel AB, Crits-Christoph P et al. The risk of depression, anxiety and suicidality in patients with psoriasis: A population-based cohort study. Arch Dermatol 2010; 146: 891-5.

5 Dalgard FJ, Gieler U, Tomas-Aragones L et al. The Psychological Burden of Skin Diseases: A Cross-Sectional Multicenter Study among Dermatological Out-Patients in 13 European Countries. J Invest Dermatol 2015; 135: 984-91.

6 Wu JJ, Penfold RB, Primatesta P et al. The risk of depression, suicidal ideation, and suicide attempt in patients with psoriasis, psoriatic arthritis, or ankylosing spondylitis. J Eur Acad Dermatol Venereol 2017; 31: 1168-75.

7 Dowlatshahi EA, Wakkee M, Arends LR et al. The Prevalence and Odds of Depressive Symptoms and Clinical Depression in Psoriasis Patients: A Systematic Review and MetaAnalysis. J Invest Dermatol 2014; 134: 1542-51.

$8 \quad$ Krueger G, Koo J, Lebwohl M et al. The impact of psoriasis on quality of life: Results of a 1998 national psoriasis foundation patient-membership survey. Arch Dermatol 2001; 137: 280-4.

9 Stern RS, Nijsten T, Feldman SR et al. Psoriasis is common, carries a substantial burden even when not extensive, and is associated with widespread treatment dissatisfaction. J Invest Dermatol Symp Proc 2004; 9: 136-9.

10 McKenna KE, Stern RS. The impact of psoriasis on the quality of life of patients from the 16center PUVA follow-up cohort. J Am Acad Dermatol 1997; 36: 388-94.

11 Pompili M, Innamorati M, Trovarelli $S$ et al. Suicide risk and psychiatric comorbidity in patients with psoriasis. J Int Med Res 2016; 44: 61-6.

12 Singhal A, Ross J, Seminog $\mathrm{O}$ et al. Risk of self-harm and suicide in people with specific psychiatric and physical disorders: comparisons between disorders using English national record linkage. J R Soc Med 2014; 107: 194-204.

13 Egeberg A, Hansen PR, Gislason GH et al. Risk of self-harm and nonfatal suicide attempts, and completed suicide in patients with psoriasis: a population-based cohort study. Br J Dermatol 2016; 175: 493-500.

This article is protected by copyright. All rights reserved. 
14 Lee M-S, Yeh Y-C, Chang Y-T et al. All-Cause and Cause-Specific Mortality in Patients with Psoriasis in Taiwan: A Nationwide Population-Based Study. J Invest Dermatol 2017; 137: 1468-73.

15 Singh S, Taylor C, Kornmehl $\mathrm{H}$ et al. Psoriasis and suicidality: A systematic review and metaanalysis. J Am Acad Dermatol 2017; 77: 425-40.

16 Chi C-C, Chen T-H, Wang S-H et al. Risk of Suicidality in People with Psoriasis: A Systematic Review and Meta-Analysis of Cohort Studies. Am J Clin Dermatol 2017; 18: 621-7.

17 Benchimol El, Smeeth L, Guttmann A et al. The REporting of studies Conducted using Observational Routinely-collected health Data (RECORD) Statement. PLoS Med 2015; 12: e1001885.

18 Khan NF, Harrison SE, Rose PW. Validity of diagnostic coding within the General Practice Research Database: a systematic review. Br J Gen Pract 2010; 60: e128-e36.

19 Herrett E, Thomas SL, Schoonen WM et al. Validation and validity of diagnoses in the General Practice Research Database: a systematic review. Br J Clin Pharmacol 2010; 69: 4-14.

20 Hennessy S, Bilker W, Berlin J et al. Factors influencing the optimal control-to-case ratio in matched case-control studies. Am J Epidemiol 1999; 149: 489.

21 Department for Communities and Local Government. English indices of deprivation: guidance. https://www.gov.uk/government/publications/english-indices-of-deprivation2010-guidance. Accessed June 302017.

22 Adelstein A, Mardon C. Suicides 1961-1974. Population Trends 02 1975: 13-8.

23 Linsley KR, Schapira K, Kelly TP. Open verdict v. suicide - importance to research. $B r \mathrm{~J}$ Psychiatry 2001; 178: 465-8.

24 Carr MJ, Ashcroft DM, Kontopantelis E et al. The epidemiology of self-harm in a UK-wide primary care patient cohort, 2001-2013. BMC Psychiatry 2016; 16: 53.

25 Springate DA, Kontopantelis E, Ashcroft DM et al. ClinicalCodes: an online clinical codes repository to improve the validity and reproducibility of research using electronic medical records. PLoS One 2014; 9: e99825.

26 Schuerch M, Gasse C, Robinson NJ et al. Impact of varying outcomes and definitions of suicidality on the associations of antiepileptic drugs and suicidality: comparisons from UK Clinical Practice Research Datalink (CPRD) and Danish national registries (DNR).

Pharmacoepidemiol Drug Saf 2016; 25: 142-55.

27 Thomas KH, Davies N, Metcalfe $\mathrm{C}$ et al. Validation of suicide and self-harm records in the Clinical Practice Research Datalink. Br J Clin Pharmacol 2013; 76: 145-57.

28 Parisi R, Rutter MK, Lunt $\mathrm{M}$ et al. Psoriasis and the Risk of Major Cardiovascular Events: Cohort Study Using the Clinical Practice Research Datalink. J Invest Dermatol 2015; 135: 2189-97.

This article is protected by copyright. All rights reserved. 
29 Qin P, Webb R, Kapur $\mathrm{N}$ et al. Hospitalization for physical illness and risk of subsequent suicide: a population study. J Intern Med 2013; 273: 48-58.

30 Nordentoft $\mathrm{M}$, Mortensen $\mathrm{P}$, Pedersen $\mathrm{C}$. Absolute risk of suicide after first hospital contact in mental disorder. Arch Gen Psychiatry 2011; 68: 1058-64.

31 Henseler T, Christophers E. Psoriasis of early and late onset: Characterization of two types of psoriasis vulgaris. J Am Acad Dermatol 1985; 13: 450-6.

32 Griffiths CEM, Christophers E, Barker JNWN et al. A classification of psoriasis vulgaris according to phenotype. Br J Dermatol 2007; 156: 258-62.

33 Parisi R, Webb RT, Carr MJ et al. Alcohol-related mortality in patients with psoriasis: A population-based cohort study. JAMA Dermatol 2017; 153: 1256-62.

This article is protected by copyright. All rights reserved. 
Table 1. Characteristics of patients at baseline in the psoriasis and psoriasis-free comparison cohorts

Psoriasis cohort

56,961

\section{Comparison cohort}

876,919

No.

\section{Demographics}

$\begin{array}{lcc}\text { Men (\%) } & 27,363(48.0) & 390,086(44.5) \\ \text { Women (\%) } & 29,598(52.0) & 486,833(55.5) \\ \text { Median age (IQR) } & 47(29) & 46(29) \\ \text { IMD score }{ }^{\dagger} \text { quintile (\%) } & & \\ \mathbf{1} \text { (least deprived) } & 13,157(23.1) & 210,938(24.1) \\ \mathbf{2} & 12,867(22.6) & 201,811(23.0) \\ \mathbf{3} & 11,402(20.0) & 174,987(20.0) \\ \mathbf{4} & 10,903(19.1) & 160,824(18.3) \\ \mathbf{5} \text { (most deprived) } & 8,569(15.0) & 127,300(14.5) \\ \text { Unknown (\%) } & 63(0.1) & 1,059(0.1)\end{array}$

† Index of Multiple Deprivation (IMD) measures area-level socioeconomic status and is divided into quintiles.

1 corresponds to the least deprived area; 5 to the most deprived area. This variable was the only variable examined with missing data ( $0.12 \%$ overall).

For this variable, a category for missing IMD quintile values was created and fitted in the multivariable model.

This article is protected by copyright. All rights reserved. 
Table 2 Psychiatric diagnostic categories and psychotropic medication prescribing in the psoriasis and psoriasis-free comparison cohorts

\begin{tabular}{lcccc} 
& Psoriasis cohort & Comparison cohort & $\begin{array}{l}\text { Prevalence Ratio } \\
\text { (95\% Cls) }\end{array}$ & p-values \\
No. & 56,961 & 876,919 & & \\
\hline Psychiatric comorbidity* & & & & \\
Schizophrenia (\%) & $511(0.9)$ & $7,842(0.9)$ & $0.94(0.86-1.03)$ & 0.206 \\
Bipolar disorder (\%) & $362(0.6)$ & $4,439(0.5)$ & $1.23(1.10-1.37)$ & $<0.001$ \\
Depression (\%) & $10,731(18.8)$ & $143,954(16.4)$ & $1.17(1.15-1.20)$ & $<0.001$ \\
Anxiety disorder (\%) & $10,187(17.9)$ & $139,663(15.9)$ & $1.15(1.13-1.18)$ & $<0.001$ \\
Eating Disorder (\%) & $472(0.8)$ & $7,007(0.8)$ & $1.08(0.98-1.18)$ & 0.119 \\
Personality Disorder (\%) & $319(0.6)$ & $4,801(0.6)$ & $0.98(0.88-1.10)$ & 0.756 \\
Any mental illness (\%) & $16,629(29.2)$ & $231,247(26.4)$ & $1.13(1.11-1.15)$ & $<0.001$ \\
Alcohol Misuse* (\%) & $1,397(2.5)$ & $15,527(1.8)$ & $1.30(1.23-1.37)$ & $<0.001$ \\
Self-harm* (\%) & $2,193(3.9)$ & $30,023(3.5)$ & $1.10(1.06-1.15)$ & $<0.001$ \\
Psychotropic medication & & & & $<$ \\
Antidepressant drugs (\%) & $16,040(28.2)$ & $213,518(24.4)$ & $1.19(1.17-1.21)$ & $<0.001$ \\
Antipsychotic drugs (\%) & $7,401(13.0)$ & $100,514(11.5)$ & $1.16(1.14-1.19)$ & $<0.001$ \\
Anxiolytics and hypnotics (\%) & $25,693(45.1)$ & $348,261(39.7)$ & $1.15(1.14-1.17)$ & $<0.001$ \\
Any psychotropic medicine (\%) & $31,024(54.5)$ & $430,500(49.1)$ & $1.13(1.12-1.14)$ & $<0.001$ \\
\hline
\end{tabular}

*Comorbidities were identified from CPRD and HES 
Table 3 Event counts and crude incidence rates of suicide and non-fatal self-harm

Suicide

Non-fatal self-harm

\begin{tabular}{lcccc}
\hline & Psoriasis cohort & Comparison cohort & Psoriasis cohort & Comparison cohort \\
\hline No. & 56,961 & 876,919 & 54,709 & 813699 \\
Follow-up in years, median (IQR) & $5.0(6.4)$ & $4.3(6.1)$ & $5.0(6.4)$ & $4.4(6.1)$ \\
Person-years & 326,049 & $4,562,721$ & 313,454 & $4,259,530$ \\
Number of suicide events (\%) & $35(0.1)$ & $679(0.1)$ & $470(0.86)$ & $5,281(0.65)$ \\
Event rate/10,000 person-years & $1.1(0.8-1.5)$ & $1.5(1.4-1.6)$ & $15.0(13.7-16.4)$ & $12.4(12.1-12.7)$ \\
$(\mathbf{9 5 \%} \mathrm{Cl}) * \quad$ & $0.7(0.6-0.8)$ & $16.1(14.2-18.1)$ & $12.9(12.4-13.3)$ \\
$\quad$ Female & $0.4(0.2-0.9)$ & $2.5(2.3-2.7)$ & $13.9(12.1-15.9)$ & $11.8(11.4-12.3)$
\end{tabular}

† The international definition of suicide was used to identify suicide cases: ICD-9 codes E950-E959 and E980-E989 (excluding E988.8) and ICD-10 codes X60-X84, Y10-Y34 (excluding Y33.9), Y87.0, Y87.2.

*Crude rates

This article is protected by copyright. All rights reserved. 
Table 4 Hazard ratios of suicide and non-fatal self-harm among patients diagnosed with psoriasis versus the unaffected comparison cohort

Stratified Cox Model

\begin{tabular}{l} 
\\
\hline Unadjusted \\
Primary analysis (adjusted by \\
IMD score)
\end{tabular}

\section{Patients with at least $1 \mathrm{GP}$ visit} per year

Excluding patients with psoriatic arthritis and adjusting for IMD score

Deaths of undetermined intent excluded from suicide definition
Suicide

Non-fatal self-harm

HR (95\% Cl)

$0.60(0.42-0.85)$

p-value

HR $(95 \% \mathrm{Cl})$

0.004

$1.16(1.05-1.29)$

p-value

$0.59(0.41-0.85)$

0.004

$1.15(1.04-1.27)$

0.003

$0.54(0.36-0.82)$

0.004

$1.10(0.98-1.22)$

0.093

$0.63(0.44-0.90)$

0.011

$1.17(1.06-1.30)$

0.002

0.008

This article is protected by copyright. All rights reserved. 
Figure 1 Hazard ratios for suicide among people with psoriasis vs. the comparison cohort according to age at diagnosis

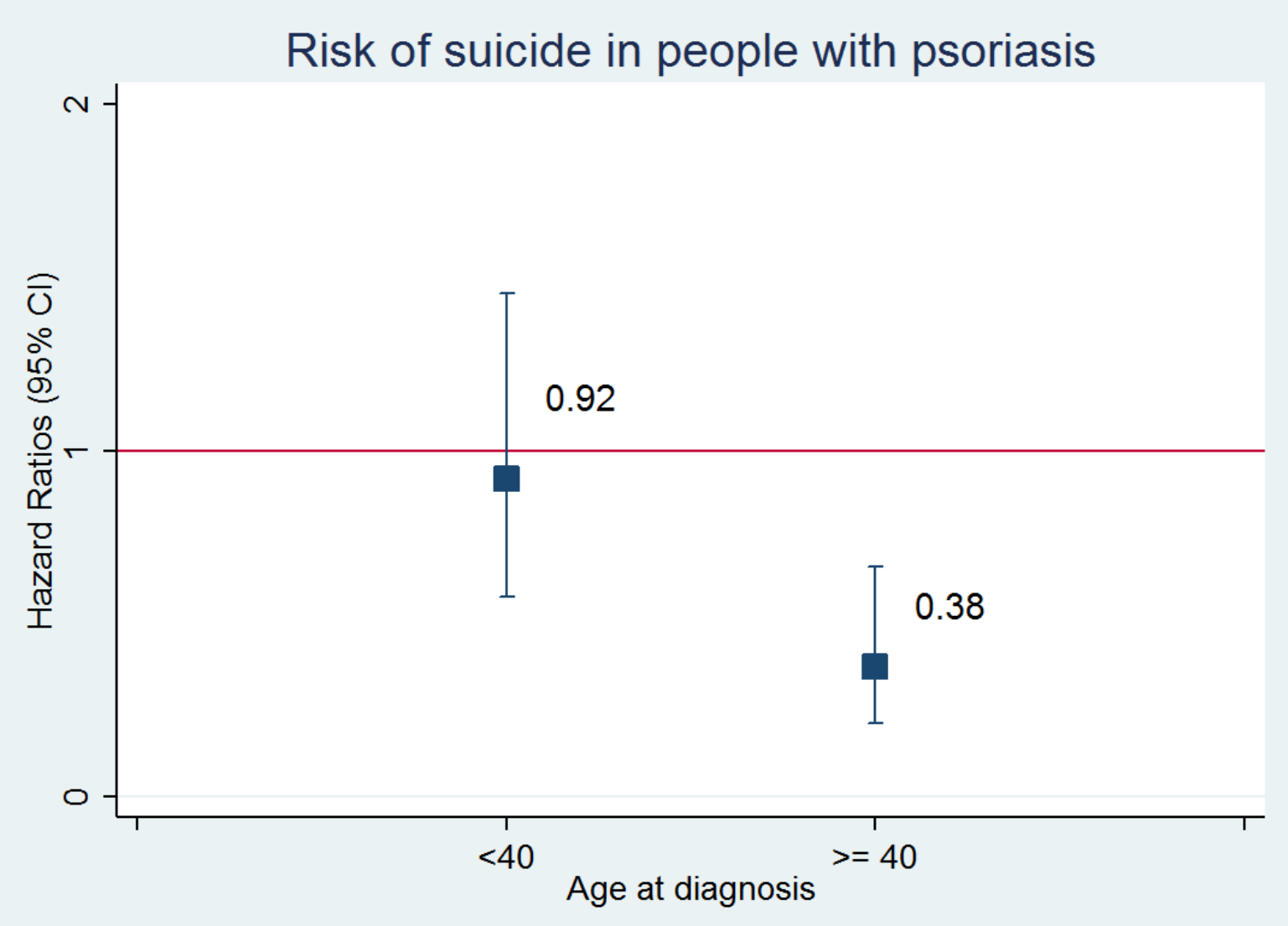

This article is protected by copyright. All rights reserved. 